UT-699

Jan. '95

\title{
Comment on a mechanism of dynamical breaking of supersymmetry
}

\author{
Tomohiro Matsuda \\ Department of Physics, University of Tokyo \\ Bunkyo-ku, Tokyo 113, Japan
}

\begin{abstract}
We re-examine the so-called Nambu-Jona-Lasinio mechanism suggested by Song, $\mathrm{Xu}$ and Chin in breaking the supersymmetry in the Wess-Zumino model and show that this mechanism cannot be justified without assuming special effects between fermions. The fermion condensation suggested by them corresponds to an unstable vacuum configuration. As a result, there is no fermion condensation and no supersymmetry breaking in the model discussed by them.
\end{abstract}

\section{Introduction}

Recently it has been discussed in a series of papers by Song, Xu and Chin [1] that spontaneous supersymmetry breaking can be realized in a chiral symmetric model without adding a Fayet-Iliopoulos or O'Raifeartaigh term. In their analysis, the so-called Nambu-Jona-Lasinio mechanism was used, and they suggest that the fermion pair condensation induces a mass gap between supersymmetric partners. If their mechanism really works, it would open many possibilities in supersymmetric models. The purpose of the present paper is to present the short comings of their argument and clarify the physical backgrounds of it. The main point is very simple: They neglected the one-loop effects 
of bosonic particles. Including these contributions correctly, we obtain the well-known one-loop effective potential, and their solution corresponds to an unstable configuration of this effective potential.

This paper is organized as follows. In section 2 we review the construction of an effective potential in the Wess-Zumino model. Then we re-examine the so-called NJL method proposed in [1] and clarify the physical backgrounds. Concluding remarks are also given in section 3 .

\section{Review of one-loop effective potential in $\mathrm{WZ}$ model}

The analysis of supersymmetry breaking in the Wess-Zumino model is as old as the modern theory of supersymmetry[2]. Using a superfield method, Fujikawa and Lang[3] constructed a one-loop effective potential for the Wess-Zumino model and discussed the stability of the supersymmetric vacuum. Many authors, for example in [4, later discussed this and related topics.

For the notational convention, we use the two-component representation : By explicitly separating the vacuum expectation values of bosonic fields, we derive the one-loop effective potential by means of the tadpole method [5] instead of the direct evaluation of it [3].

The starting Wess-Zumino Lagrangian for a chiral super multiplet is given by

$$
\begin{aligned}
L & =\left.\Phi^{+} \Phi\right|_{\theta \theta \overline{\theta \theta}}+\left[\left.\frac{1}{3 !} \lambda \Phi^{3}\right|_{\theta \theta}+\left.\frac{1}{2} m \Phi^{2}\right|_{\theta \theta}+\text { h.c. }\right] \\
& =i \partial_{m} \bar{\psi} \bar{\sigma}^{m} \psi+\bar{A} \square A+\bar{F} F+\left[\frac{\lambda}{2}\left(A^{2} F-\psi \psi A\right)+m\left(A F-\frac{1}{2} \psi \psi\right)+\text { h.c. }\right]
\end{aligned}
$$

Shifting the bose fields of the theory in the fashion

$$
\begin{aligned}
& A \rightarrow A+a \\
& F \rightarrow F+f
\end{aligned}
$$

we obtain

$$
\begin{aligned}
L^{\prime}= & i \partial_{m} \bar{\psi} \bar{\sigma}^{m} \psi+\bar{A} \square A+\bar{F} F \\
& +\left[\eta\left(A F-\frac{1}{2} \psi \psi\right)+\frac{\lambda}{2}(A A F-\psi \psi A)+\frac{\lambda}{2} f A A\right. \\
& \left.+F\left(m a+\frac{\lambda}{2} a^{2}-\bar{f}\right)+A \eta f+\text { h.c. }\right]
\end{aligned}
$$


here we set

$$
\eta=m+\lambda a
$$

Before calculating the effective potential, we should derive the propagators of the theory. Extracting the quadratic part of the boson fields,

$$
\begin{aligned}
& S_{0}= \int d^{4} x \frac{1}{2} \Phi^{T} A \Phi+\Phi^{T} J \\
&\left\{\begin{array}{l}
\Phi^{T}=(A, \bar{A}, F, \bar{F}) \\
J=(J, \bar{J}, K, \bar{K})
\end{array}\right. \\
& A=\left(\begin{array}{llll}
-\lambda f & \square & -\eta & 0 \\
\square & -\lambda \bar{f} & 0 & \bar{\eta} \\
-\eta & 0 & 0 & 1 \\
0 & -\bar{\eta} & 10 &
\end{array}\right)
\end{aligned}
$$

the matrix $A$ is easily inverted to obtain

$$
A^{-1}=\frac{1}{\Delta}\left(\begin{array}{llll}
\lambda \bar{f} & \square-\bar{\eta} \eta & \bar{\eta}(\square-\bar{\eta} \eta) & \lambda \bar{f} f \\
\square-\bar{\eta} \eta & \lambda f & \lambda f \bar{\eta} & \eta(\square-\bar{\eta} \eta) \\
\bar{\eta}(\square-\bar{\eta} \eta) & \lambda f \bar{\eta} & \lambda f \bar{\eta} \eta & -\lambda^{2} \bar{f} f+\square(\square-\bar{\eta} \eta) \\
\lambda \bar{f} \eta & \eta(\square-\bar{\eta} \eta) & -\lambda^{2} \bar{f} f+\square(\square-\bar{\eta} \eta) & \lambda \bar{f} \eta \eta
\end{array}\right)
$$

where

$$
\Delta=(\square-\bar{\eta} \eta)^{2}-\lambda^{2} \bar{f} f
$$

The tree level generating functional is now given by

$$
\ln Z_{0}=-\frac{i}{2} \int d^{4} x\left(J^{T} A^{-1} J\right)
$$

Looking at $\left.\frac{\delta^{2} \ln \left(Z_{0}\right)}{\delta J_{1} \delta J_{2}}\right|_{J=0}$ the propagators of the theory are obtained directly.

Now let us derive the effective potential by means of the tadpole method. According to ref. [5], the following relation exists between the derivative of effective potential and 1PI tadpole.

$$
\frac{d V\left(\phi_{0}\right)}{d \phi_{0}}=-\Gamma^{\prime(1)}
$$

In this expression $\phi_{0}$ means the vacuum expectation value(vev) of the field $\phi$ which can be any scalar field of the theory( in the present theory $\phi$ means $A$ or $F$, and $\phi_{0}$ means 
$a$ or $f), \Gamma^{\prime(1)}$ is the 1PI tadpole that is calculated after separating the vev and quantum fluctuation of the scalar fields as $\phi \rightarrow \phi+\phi_{0}$. So we use (2.3) to calculate $\Gamma^{\prime(1)}$. Using these relations, we obtain

$$
\frac{V_{0}}{d f}=m a+\frac{\lambda}{2} a^{2}-\bar{f}
$$

and

$$
\frac{d V_{1}}{d f}=-\frac{1}{2} \lambda^{2} \int \frac{d^{4} p}{(2 \pi)^{4}} \frac{\bar{f}}{\left(p^{2}+\bar{\eta} \eta\right)^{2}-\bar{f} f \lambda^{2}} .
$$

After integration we get

$$
V_{0}=\left(m a+\frac{1}{2} \lambda a^{2}\right) f-\bar{f} f+P(\bar{f}, a, \bar{a})
$$

and

$$
V_{1}=\frac{1}{2} \int \frac{d^{4} p}{(2 \pi)^{4}} \ln \left[\left(p^{2}+\bar{\eta} \eta\right)^{2}-\lambda^{2} \bar{f} f\right]+H(\bar{a}, a)
$$

where $P(\bar{f}, a, \bar{a})$ and $H(\bar{a}, a)$ are integration constants. We can impose supersymmetric boundary condition

$$
\left.V_{0}\right|_{f=0}=0
$$

and

$$
\left.V_{1}\right|_{f=0}=0
$$

Then we recover the effective potential, which is also directly calculated in ref. [3],

$$
V_{0}=\left[\left(m a+\frac{1}{2} \lambda a^{2}\right) f+h . c .\right]-\bar{f} f
$$

and

$$
V_{1}=\frac{1}{2} \int \frac{d^{4} p}{(2 \pi)^{4}} \ln \left[1-\frac{\lambda^{2} \bar{f} f}{\left(p^{2}+\bar{\eta} \eta\right)^{2}}\right] .
$$

The vacuum stability of this potential is well analyzed in ref. [3, 4. Eq.(2.17) can be evaluated as

$$
\begin{aligned}
V_{1}= & \frac{\pi^{2}}{(2 \pi)^{4}}\left\{-\frac{1}{2} \lambda^{2}|f|^{2}\left(\ln \Lambda^{2}+\frac{1}{2}\right)\right. \\
& \left.+\frac{1}{2}|f|^{2} \ln |\eta|^{2}+\frac{1}{2}|\eta|^{2}\left[\left(1-x^{2}\right) \ln (1-x)+(1-x)^{2} \ln (1+x)\right]\right\} \\
& -(Z-1)|f|^{2}
\end{aligned}
$$

where we set $x=\frac{|\lambda f|}{|\eta|^{2}}$, and $\Lambda$ stands for the ultra-violet cut-off. 
We also added the wave function renormalization factor $Z$ (in the last term in (2.18)) in order to absorb the infinity contained in $\log \Lambda^{2}$. In order to avoid the infrared singularity, which can appear because we set $m=0$ in the next section, we renormalize the wave function at

$$
|f|=0 \quad \text { and } \quad|\eta|=M
$$

where $M$ has the dimensions of a mass. The wave-function renormalization factor is then fixed as

$$
Z=1-\alpha\left(\ln \frac{\Lambda}{M^{2}}-1\right)
$$

The total effective potential up to one-loop level is now given by

$$
\begin{aligned}
V_{\text {eff }}= & -|f|^{2}\left(1-\alpha \ln \frac{|\eta|^{2}}{M^{2} \lambda^{2}}\right) \\
& +\frac{\alpha|\eta|^{4}}{2 \lambda^{2}}\left[(1+x)^{2} \ln (1+x)+(1-x)^{2} \ln (1-x)-3 x^{2}\right] \\
& +\lambda\left[\left(a_{1}^{2}-a_{2}^{2}\right) f_{1}+2 a_{1} a_{2} f_{2}\right]+2 m\left(a_{1} f_{1}+a_{2} f_{2}\right) .
\end{aligned}
$$

Here we set

$$
\begin{aligned}
& \left\{\begin{array}{c}
f=f_{1}+i f_{2} \\
a=a_{1}-i a_{2}
\end{array}\right. \\
& \alpha=\frac{\pi^{2} \lambda^{2}}{2(2 \pi)^{4}}, x=\frac{|\lambda f|}{|\eta|^{2}} .
\end{aligned}
$$

In order to discuss the vacuum stability, we parametrize $f_{1}$ and $f_{2}$ by

$$
\tan \beta=\frac{f_{1}}{f_{2}}
$$

and evaluate $V_{\text {eff }}$ at $\frac{\partial V_{\text {eff }}}{\partial \beta}=0$ ( This corresponds to the direction of the valley of the effective potential). We then find

$$
\begin{aligned}
V_{\text {eff }}= & -\frac{|\eta|^{4} x^{2}}{\lambda^{2}}\left(1-\alpha \ln \frac{|\eta|^{2}}{M^{2}}\right)+\frac{\alpha}{2}|\eta|^{4}\left[(1+x)^{2} \ln (1+x)+(1-x)^{2} \ln (1-x)-3 x^{2}\right] \\
& +\frac{x|a||\eta|^{2}}{\lambda} \sqrt{\lambda^{2}\left(a_{1}^{2}+a_{2}^{2}\right)+2 m \lambda a_{1}+m^{2}}
\end{aligned}
$$

To take account of the two possible signs of the square-root, we extend the range of $x$ to $-\infty<x<+\infty$. This potential develops an imaginary part for $|x|>1$ and this means that the solution

$$
|f| \neq 0 \quad \text { and } \quad|\eta|=0
$$


is dynamically unstable. We can find the stationary value of this effective potential in the region $|x| \leq 1$ assuming that $\alpha$ is small. The effective potential can be written as

$$
\begin{aligned}
V_{e f f} \cong & -\frac{|\eta|^{4} x^{2}}{\lambda^{2}}\left(1-\alpha \ln \frac{|\eta|^{2}}{M^{2}}\right) \\
& +\frac{x|a||\eta|^{2}}{\lambda} \sqrt{\lambda^{2}\left(a_{1}^{2}+a_{2}^{2}\right)+2 m \lambda a_{1}+m^{2}} .
\end{aligned}
$$

Taking the minimum of the potential $\left(\partial V_{e f f} / \partial x=0\right)$, we obtain

$$
V_{e f f}=\frac{|a|^{2}}{4} \frac{\lambda^{2}\left(a_{1}^{2}+a_{2}^{2}\right)+2 m \lambda a_{1}+m^{2}}{1-\alpha \ln \frac{|\eta|^{2}}{M^{2}}}
$$

for

$$
x=\frac{1}{2} \frac{\lambda|a| \sqrt{\lambda^{2}\left(a_{1}^{2}+a_{2}^{2}\right)+2 m \lambda a_{1}+m^{2}}}{|\eta|^{2}\left(1-\alpha \ln \frac{|\eta|^{2}}{M^{2}}\right)}
$$

This potential has its minimum at

$$
a_{1}=0, \quad a_{2}=0, \text { and } f=0
$$

or

$$
a_{1}=-\frac{m}{\lambda}, \quad a_{2}=0, \text { and } f=0
$$

In both solutions, $f$ is zero and supersymmetry is not broken. The second solution gives non-zero vev of $a$ but $f$ still remains zero: Two solutions (2.28) are actually two stable physically equivalent solutions, since one can pass from one to the other by a redefinition of the fields 2]. When we consider the massless Wess-Zumino model in the next section, the second solution becomes $a_{1}=0, a_{2}=0$ so the vev of $a$ remains zero. Detailed study of this phenomenon from another point of view is given in ref. [2].

Let us examine the physical meanings of this solution. At the tree level, the equation of motion for auxiliary field is

$$
F=\frac{1}{2} \lambda \bar{A}^{2}
$$

At the first glance, this equation seems to suggest that if the tree level potential develops a non-zero vacuum expectation value $\langle\bar{A}\rangle,\langle F\rangle$ becomes non-zero and the supersymmetry of the theory can be broken spontaneously. But this does not happen. Including higher order quantum corrections, supersymmetry-breaking vacuum $\left(<F>=\frac{1}{2} \lambda<\bar{A}>^{2}\right.$ 
and $<\bar{A}>$ is non-zero) becomes unstable and the supersymmetric vacuum $(<F>=0)$ remains stable.

Furthermore, there is no $\Lambda$ dependence in the effective potential after renormalization of the wave-function.

To analyze the behavior of the effective potential at small $|\eta|$ reliably, the renormalization group improvement of the effective potential has also been discussed in ref. [3]. The effective potential for the massless theory is

$$
\begin{aligned}
V_{\text {eff }}= & -\frac{|\eta|^{4} x^{2}}{\lambda^{2}}\left(1-\alpha \ln \frac{|\eta|^{2}}{M^{2}}\right)+\frac{\alpha}{2}|\eta|^{4}\left[(1+x)^{2} \ln (1+x)+(1-x)^{2} \ln (1-x)-3 x^{2}\right] \\
& +x|a|^{2}|\eta|^{2}
\end{aligned}
$$

The stationary value of this potential in this region $|x|<1$ is estimated to be

$$
V_{e f f}=\frac{\lambda^{2}|a|^{4}}{4\left(1-\alpha \ln \frac{|\eta|^{2}}{M^{2}}\right)}
$$

at

$$
x=\frac{\lambda|a|^{2}}{|\eta|^{2}\left(1-\alpha \ln \frac{|\eta|^{2}}{M^{2}}\right)} .
$$

Renormalization group improvement of $V_{\text {eff }}$ suggests that

$$
\begin{aligned}
V_{e f f} & \simeq \frac{1}{4}\left(\lambda(M)|a|^{3}\right)^{\frac{4}{3}} \lambda(|a|)^{\frac{2}{3}} \\
& \simeq \frac{1}{4}(\lambda(M))^{2}|a|^{4} \frac{1}{\left(1-3 \alpha \ln \frac{|\eta|^{2}}{M^{2}}\right)^{\frac{1}{3}}}
\end{aligned}
$$

with the running coupling

$$
\lambda(|a|)=\frac{\lambda(M)}{\left[1-3 \alpha \ln \frac{|a|^{2}}{M^{2}}\right]^{\frac{1}{2}}} .
$$

Note that the combination $\lambda(M)|a|^{3}$ is renormalization group invariant in this theory.

$V_{\text {eff }}$ in (2.33) has a minimum at $|a|=0$ for which $\lambda(|a|) \rightarrow 0$ and the analysis of $V_{\text {eff }}$ is reliable. For $|a| \rightarrow 0, x \rightarrow 0$ in (2.33) and thus $|f| \rightarrow 0$ and no supersymmetry breaking.

This explicit analysis, which is useful to the discussion in the next section, is of course consistent with the analysis on the basis of Witten index 6].

For the discussion of the next section, we summarize the results restricting ourselves to the massless Wess-Zumino model. First, there is no supersymmetry-breaking vacuum. Second, the vev of scalar field $A$ remains zero. 


\section{The meaning of NJL method in WZ model}

In this section we re-examine the physical backgrounds of the NJL method proposed in ref. [1]. For convenience, we first recapitulate the basic procedure in ref. [1].

The same lagrangian (2.1) is used, but at the first stage we eliminate the auxiliary field $F$ using the equation of motion. The result is (we here set $m=0$ )

$$
L=i \partial_{m} \bar{\psi} \bar{\sigma}^{m} \psi+A^{*} \square A-\left[\frac{\lambda}{2} \psi \psi A+\text { h.c. }\right]-\frac{1}{4} \lambda^{2}|A|^{4} .
$$

The equations of motion are given by

$$
\left\{\begin{array}{cc}
\square A+\frac{1}{2} \lambda^{2} A^{*} A A+\frac{1}{2} \lambda \overline{\psi \psi} & =0 \\
\square A^{*}+\frac{1}{2} \lambda^{2} A^{*} A^{*} A+\frac{1}{2} \lambda \psi \psi & =0 \\
{\left[i \partial_{m} \bar{\sigma}^{m}-\lambda A\right] \psi} & =0
\end{array}\right.
$$

Taking the vacuum expectation value of the first equation in (3.2), one obtains

$$
<A>+\frac{1}{2} \lambda^{2}<A A A^{*}>=-\frac{1}{2} \lambda<\overline{\psi \psi}>
$$

Expansion of $<A^{*} A A>$ and $<\overline{\psi \psi}>$ to the one-loop level(i.e., to the order of $\hbar$ ) is given by

$$
\left\{\begin{array}{l}
<A^{*} A A>=<A^{*}><A><A>+<A^{*}>[- \text { AAloop }]+<A>\left[-A A^{*} \text { loop }\right] \\
<\overline{\psi \psi}>=[\overline{\psi \psi} \text { loop }] .
\end{array}\right.
$$

Here the results of the one-loop diagrams are symbolically represented. Then eq.(3.3) becomes, to the one-loop order,

$$
\begin{aligned}
0= & \square a+\frac{\lambda^{2}}{2} a a a^{*} \\
& +\frac{\lambda^{2}}{2} a\left[-A A^{*} \text { loop }\right]+\frac{\lambda^{2}}{2} a^{*}[- \text { AAloop }]+\frac{\lambda}{2}[-\overline{\psi \psi} \text { loop }]
\end{aligned}
$$

Neglecting the tadpoles of the bosonic fields and setting $\square a=0$ in (3.5), we get the same answer as in ref. [1];

$$
\lambda a a a^{*}+\operatorname{Tr}\left[\frac{\lambda}{2} \int \frac{d^{4} p}{(2 \pi)^{4}} \frac{1}{i \partial_{m} \bar{\sigma}^{m}-\lambda a^{*}}\right]=0 .
$$


which leads to the fermion pair condensation and a mass gap between the supersymmetric partners [1]. In fact, the above equation (3.6) can be rewritten as

$$
|a|^{2}=4 \int \frac{d^{4} p}{(2 \pi)^{4}} \frac{1}{p^{2}-\lambda^{2}|a|^{2}}
$$

This equation looks like a well-known mass-gap equation. The integration requires an ultra-violet cut-off, so the solution $(a)$ of the self-consistent equation(3.7) depends on the ultra-violet cut-off parameter. Shifting the fields in the lagrangian as $A \rightarrow A+a$, with $a$ given by eq.(3.7), we obtain the masses

$$
\begin{aligned}
& m_{A}^{2}=\frac{\lambda^{2}}{2}|a|^{2} \\
& m_{\psi}=\lambda|a| .
\end{aligned}
$$

The supersymmetric partners thus appears to acquire different masses. This is the mechanism noted in ref. [1].

But we must not neglect bosonic tadpoles. As discussed in the previous section, the neglect of bosonic tadpoles in (3.5) is not consistent with the expansion in $\hbar$ and the resulting effective potential corresponds to the expansion around an unstable vacuum (i.e., $x=\frac{1}{2}$ in $(2.23)$ ). The meaning of the equation (3.3) is now clear: This equation means that the derivative of the effective potential is set to zero at the minimum, i.e., $\left.\frac{\partial\left(V^{0}+V^{\text {one-loop }}\right)}{\partial a^{*}}\right|_{v a c}=0$. One can easily obtain (3.3) by applying the tadpole method (2.9) to the variable $a$, not to $f$. Substituting $A$ in (3.1) as $A \rightarrow A+a$ and using the tadpole method, one obtains

$$
\begin{aligned}
\frac{d\left(V_{0}+V_{1}\right)}{d a^{*}}= & \frac{\lambda^{2}}{2} a a a^{*} \\
& +\frac{1}{2} \lambda[-\overline{\psi \psi} \text { loop }]+\frac{1}{2} \lambda^{2} a\left[-A A^{*} \text { loop }\right]+\frac{1}{2} \lambda^{2} a^{*}[- \text { AAloop }]
\end{aligned}
$$

The evaluation and integration of (3.9) is slightly complicated in the present calculational scheme but the result is the same as (2.30)(see ref.[四). Of course, there is no cutoff dependence in the final result which explicitly remains in the analysis of ref. [1], nor supersymmetry breaking induced by fermion pair condensation in the full effective potential resulting from (3.9). The stationary point of the effective potential correspond to the supersymmetry preserving point of (2.30). 
In conclusion, we have shown that the supersymmetry breaking solution in ref.[1] is a direct consequence of the neglect of one-loop bosonic effects in the loop expansion of the effective potential. Since no dynamical mechanism why the one-loop fermion effects should be retained and why the one-loop boson effects should be neglected is given in ref. [1], we conclude that the so-called Nambu-Jona-Lasinio mechanism suggested there is

not justified in the conventional framework of field theory without assuming some special attractive force between fermions.

\section{Acknowledgment}

We thank K.Fujikawa and A.Yamada for many helpful discussions.

\section{References}

[1] He-Shan Song and Guang-Nan Xu, J. Phys. A26 (1993) 2699

He-Shan Song, Guang-Nan Xu and Ying An Chin, J. Phys. A26 (1993) 4463

He-Shan Song and Guang-Nan Xu, J. Phys. A25 (1992) 4941

[2] J.Iliopoulos and B.Zumino, Nucl.Phys.B76(1974)310

[3] K.Fujikawa and W.Lang, Nucl.Phys.B88(1975)77

[4] R.Miller, Phys.Lett.124B(1983)59, Nucl.Phys.B241(1984)535

[5] S.Weinberg, Phys.Rev.D7(1973)2887

[6] E.Witten, Nucl.Phys. B202(1982)253 